\title{
Metatarsal Fracture Reduction and Fixation by Kirschner Wires: Technical Tip
}

\author{
Eli Peled*, Doron Norman, Eyal Melamed \\ Department of Orthopaedics B, Rambam Health Care Campus, and The Bruce Rappaport \\ Faculty of Medicine, Technion-Israel Institute of Technology, Haifa, Israel \\ Email: ${ }^{*}$ _peled@rambam.health.gov.il
}

Received March 12, 2012; revised April 20, 2012; accepted April 28, 2012

\begin{abstract}
The incidence of metatarsal fractures is not precisely known. Malposition might cause future metatarsalgia, mid-foot pain and discomfort, which may dictate an on-going need for in-shoe orthosis, shoe modification and, occasionally, operative correction. To minimize and prevent these problems, we describe a simple technique for reduction and fixation of metatarsal fractures by Kirschner wires. We have found this technique to be a simple and efficient way of reducing and fixing metatarsal fractures. It is applied easily by the closed and open technique.
\end{abstract}

Keywords: Fracture Reduction; Kirschner Wire; Metatarsal Fixation; Metatarsal Fracture; Metatarsal Reduction

\section{Introduction}

The precise incidence of metatarsal fractures is unknown; most of the literature describes stress fractures, especially among young athletes and military recruits. Hasselman et al found that the incidence of foot fracture among old women is $3 / 1000$, most involving the fifth metatarsal [1]. Malunion of these fractures might be a cause of future metatarsalgia, which may dictate a continuous need for in-shoe orthosis, shoe modification and occasionally operative correction [2,3]. Other complications of metatarsal fractures include loss of reduction, delayed union and avascular necrosis of the metatarsal head. Accurate reduction and stable fixation is the key for favorable results in some of the displaced fractures.

Fracture reduction can be achieved by closed or open means, and fixation might be used by plating, intramedullary screws, tension-band wiring, mini external fixation, and intramedullary Steinmann pin [2-8]. Nevertheless, Kirschner's wires (KW) are the most commonly used fixation device; they may be introduced in a closed percutaneous technique or by an open mode as part of open reduction and internal fixation surgery. In such a case they are often introduced using the "antegrade-retrograde" technique. In this technique the KW is introduced into the distal metatarsal shaft and/or neck through the fracture, out of the metatarsal head (usually throughout the sole of the foot). The KW is then grabbed by the drill at the sole of the foot, the fracture is reduced and the $\mathrm{KW}$ is driven retrograde to the base of the metatarsus.

\footnotetext{
*Corresponding author.
}

The KW needs to hold in the base of the metatarsus for a stable fixation, but not penetrate into the Lisfranc joint. This is sometimes not easy, especially in osteoporotic patients. The advantage of intramedullary fixation by $\mathrm{KW}$ is its relatively simple application, minimal or no periosteal stripping, and easy removal. This technique is especially appealing when the nature of the fracture and the degree of comminution precludes distal or proximal fixation of plates or screws (e.g., metatarsal neck fractures) [8]. Although it is usually not as stable as other internal fixation devices, the stability secured by $\mathrm{KW}$ suffices due to the inherent stability of the metatarsus, which is stabilized by the neighboring metatarsals.

We describe a simple technique for reduction and fixation to prevent penetration into the Lisfranc joint by the Kirschner wires.

\section{Technique}

After preparing the metatarsal fracture site for an open reduction (Figures 1(a), (b)), a Kirschner wire (KW) which has one sharp and one blunt end is used for intramedullary fixation. It is best introduced free hand in an antegrade fashion from the fracture into the medullary canal of its distal segment. Introducing the KW by the free-hand technique allows more sensitive palpation of the fracture site. Advancing the $\mathrm{KW}$ in the canal helps to achieve the desired location with extensive fluoroscopy use. Intraoperative images are necessary to confirm the correct location of the KW. Once the correct position of the KW within the canal of the distal fragment is con- 
firmed, the distal part is pushed against the head and helps to bring the metatarsus into length. At times, especially when the fracture is not fresh, significant force is necessary to overcome the shortening. In such a case, two maneuvers can be done: After mounting the KW on a drill, the drill and the KW are pushed forcibly without
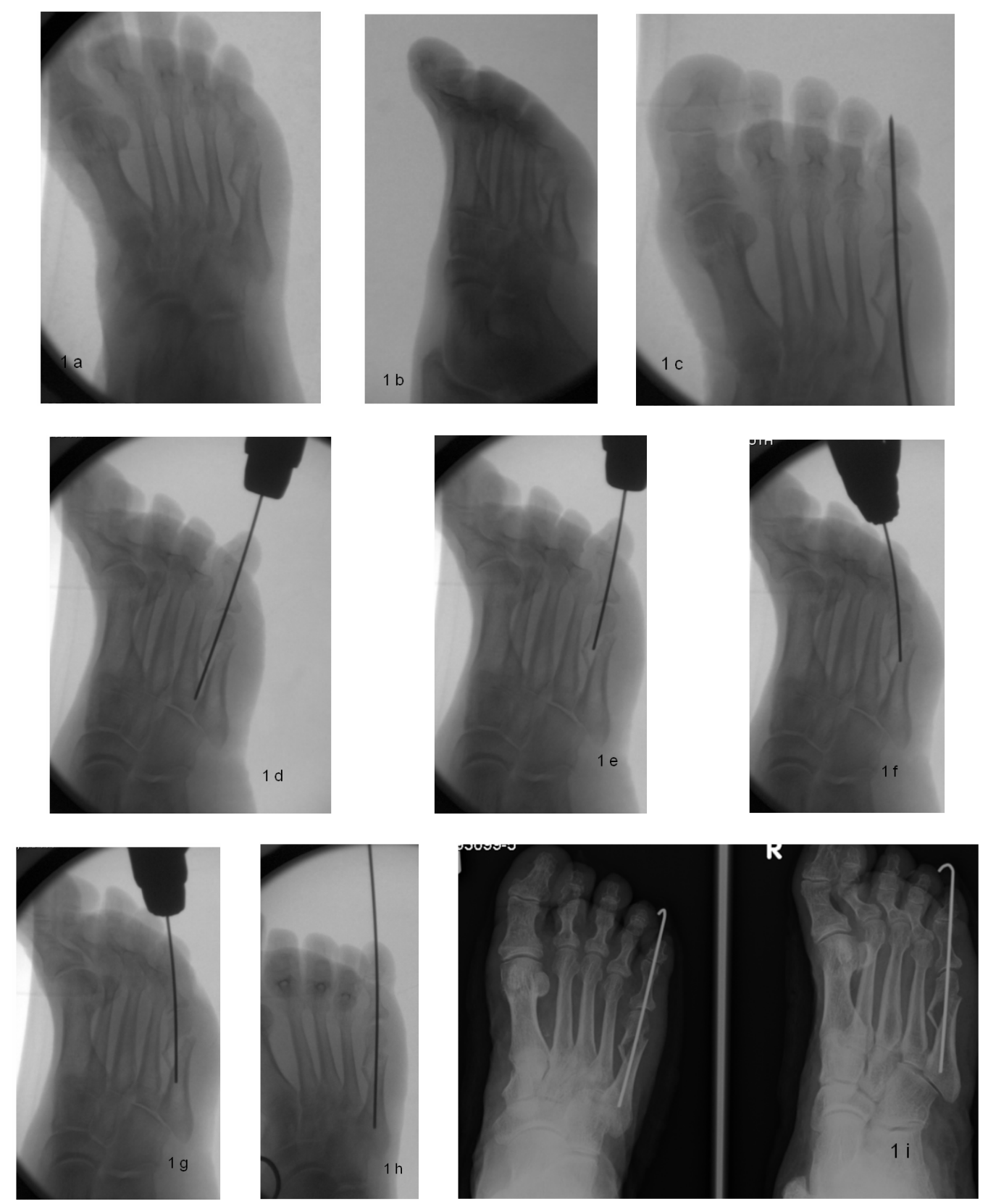

Figure 1. Percutaneous closed reduction and internal fixation of fifth metatarsal fracture with shortening and mild comminution in an 80-year old woman (a, b); A Kirschner wire (KW) has been introduced through the fracture into the distal fragment and the base of the proximal phalanx. The KW sharp tip is protruding out through the plantar skin (c); The drill is mounted on the distal sharp end of the $\mathrm{KW}$ (d); which is pulled back until the proximal end is at the level of the fracture (e); An attempt is made to redirect the dull proximal end of the $\mathrm{KW}$ into the canal of the proximal part of the metatarsus (f, g); After the KW location within the proximal fragment medullary canal was verified in two plane fluoroscopy. It was pushed and drilled proximally until its dull end abutted against the base of the metatarsus (h); There is increased resistance to further advancement of the $\mathrm{KW}$, which is used for further reduction and length restoration by pushing the drill and, at the same time, pulling the toe. In osteoporotic patients, this maneuver should be carried out cautiously, lest penetration into the joint occurs. The distal tip of the $\mathrm{KW}$ is cut and bent. Two weeks post surgery, radiography demonstrate good location of the $\mathrm{KW}$ (i); Length has been only partly restored due to the closed nature of the surgery, the two weeks that has elapsed from the injury to the surgery, and the osteoporotic bone which precluded forceful reduction. 
rotating the KW. In this way a pushing force rather than a drilling one is applied manually. If more distraction force is needed, the toe can be grabbed from both sides at the proximal phalanx with a small Webber clamp or a towel clamp and pulled. However, the advantage of directly pushing on the head in terms of less harm to the toe is obvious.

Once the desired possible length has been achieved, the KW is drilled through the metatarsal head into the base of the proximal phalanx of the toe with its sharp edge exiting from the sole (Figure 1(c)). We recommend keeping the metatarsophalangeal joint in a slight plantarflexed position, as this joint not infrequently ends up in dorsal subluxation due to scarring that occurs to the dorsal elements and the extensor tendons as a result of the injury. The drill is disengaged from the proximal part of the KW and is assembled on its distal part in the sole of the foot (Figure 1(d)). The wire is pulled to the level of the fracture (Figure 1(e)). Open or closed reduction of the fracture is achieved by pushing the proximal part of the $\mathrm{KW}$ into the proximal intramedullary canal (Figures 1(f), (g)). If done in a closed fashion, fluoroscopy is used to assess the position of the $\mathrm{KW}$ within the canal in two planes. The KW is drilled further within the metatarsal medullary canal until it stops at the metatarsal base which exerts increased resistance on the dull proximal end of the KW (Figure 1(h)). Gentle but forceful pushing with the drill rotating and at the same time pulling on the toe will return some additional length. Although the $\mathrm{KW}$ has a blunt edge that stops at the base of the metatarsus, forceful pushing of the running drill may cause penetration if osteoporotic bone is involved. The wire end protruding from the sole is cut and bent, leaving a small edge for future removal (Figure 1(i)).

\section{Results and Conclusion}

We have found this technique to be a simple and easy way for reducing and fixing metatarsal fractures. It can be applied by closed or open techniques, preventing additional damage to the soft tissue surrounding the bone.

\section{REFERENCES}

[1] C. Hasselman, M. T. Vogt, K. L. Stone, J. A. Cauley and S. F. Conti, "Foot and Ankle Fractures in Elderly White Women: Incidence and Risk Factors," Journal of Bone and Joint Surgery American, Vol. 85, No. 5, 2003, pp. 820-824.

[2] R. A. Mann and L. B. Chou, "Surgical Management for Intractable Metatarsalgia,” Foot and Ankle International, Vol. 16, No. 6, 1995, pp. 322-327.

[3] A. E. Sanchez, C. V. Vicent, P. Alcantara and A. J. Llabres, "Fractures or the Central Metatarsal," Foot and Ankle International, Vol. 17, No. 4, 1996, pp. 200-203.

[4] H. J. Aguado, P. G. Herranz and J. M. Rapariz, "Metaizeau's Technique for Displaced Metatarsal Neck Fractures,” Journal of Pediatric Orthopedics B, Vol. 12, No. 5, 2003, pp. 350-353. doi:10.1097/01202412-200309000-00011

[5] B. D. Owens, J. J. Wixted, J. Cook and A. K. Teebagy, "Intramedullary Transmetatarsal Kirschner Wire Fixation of Lisfranc Fracture-Dislocations," American Journal of Orthopedics, Vol. 32, No. 8, 2003, pp. 389-391.

[6] J. Sarimo, J. Rantanen, S. Orava and J. Alanen, “TensionBand Wiring for Fractures of the Fifth Metatarsal Located in the Junction of the Proximal Metaphysis and Diaphysis,” American Journal of Sports Medicine, Vol. 34, No. 3, 2006, pp. 476-480. doi:10.1177/0363546505281803

[7] T. A. Schildhauer, S. E. Nork and B. J. Sangeorzan, "Temporary Bridge Plating of the Medial Column in Severe Midfoot Injuries,” Journal of Orthopedic Trauma, Vol. 17, No. 7, 2003, pp. 513-520. doi:10.1097/00005131-200308000-00007

[8] E. J. Verzin and S. A. Henderson, "A New Technique for the Management of Difficult Metatarsal Neck Fractures," Foot and Ankle International, Vol. 21, No. 10, 2000, pp. 868-869. 